América sin Nombre, n. ${ }^{\circ} 23$ (2018): 17-23

DOI 10.14198/AMESN.2018.23.00

ISSN: 1577.3442 / eISSN: 1989-9831
Modo de citación de este artículo:

Higashi, Alejandro y Ballester, Ignacio. «Presentación: Ser poeta joven en México". Madurez de la joven poesía mexicana. Alejandro Higashi e Ignacio Ballester (coordinadores). América sin Nombre, 23 (2018): 17-23, DOI: 10.14198/AMESN.2018.23.00

Link para este artículo: http://dx.doi.org/10.14198/AMESN.2018.23.00

\title{
Presentación Ser poeta joven en México
}

\author{
Presentation \\ Be a young poet in Mexico
}

Alejandro Higashi

Universidad Autónoma Metropolitana, Unidad Iztapalapa I Academia Mexicana de la Lengua

IGNACIO BALLESTER

Universidad de Alicante

Ya somos todo aquello contra lo que luchamos a los veinte años José Emilio Pacheco, «Antiguos compañeros se reúnen» Desde entonces, 1980.

No soy un poeta joven pero lo fui alguna vez Julián Herbert, «Autorretrato a los 41» Álbum Iscariote, 2013.

"Joven» es un término problemático, aunque no tanto como «viejo». El tiempo condiciona los estudios críticos; también el espacio. Con Madurez de la joven poesía mexicana nos referimos a quienes están publicando poesía en el aquí y en el ahora de México. Con ahora hacemos alusión al siglo Xxi y con aquí a un país que enfrenta día a día crisis económicas, de seguridad, de educación, de ingobernabilidad y muchas otras, sin rendirse. La mayoría de edad del tercer milenio nos permite ubicar a poetas que nacen a partir de los setenta y que publican en el umbral de la nueva centuria; esas voces que recibían premios y estímulos para jóvenes creadores, y hoy son garantes ya de una poética consolidada, tienen varios libros publicados que están llamados a definir distintos hitos de estas promociones y cuya obra es ya objeto de estudios críticos (aunque esto último todavía no sea tan común).

La poesía mexicana es particular por varios motivos: aún recuerda a Octavio Paz, como veremos; reúne gran parte de las experimentaciones formales que se dan en el ámbito hispanoamericano, según lo Sustituir por explica Carmen Alemany (2009); y da pie a continuas polémicas por el dinero que el Estado invierte en ella. Dicho apoyo estatal, que debería de ser una virtud, una ventaja en el ámbito de la cultura internacional, provoca, por ejemplo, que existan poetas jóvenes hasta los treinta y cinco años y que dicha franja sea tan amplia que incluso debe subdividirse en tres categorías básicas: joven creador con poca o nula trayectoria, joven creador con trayectoria A y joven creador con trayectoria B (Rueda Olvera 
19). Después de esa franja etaria, las convocatorias públicas del Fondo Nacional para la Cultura y las Artes (FONCA) se refieren simplemente a creadores.

Más allá del reflejo institucional que define quién es joven y quién no, se encuentra un grupo de entusiastas de la poesía que escriben en una edad bisagra entre el talento en potencia (que les auguraba numerosos galardones, un lugar de honor en las antologías y muchos títulos en editoriales prestigiosas) y un oficio, el poético, del que, como sabemos, es difícil vivir. Se trata de autores y autoras que influyen sobre la obra de las generaciones más recientes, nacidas hacia la década de 1990, y que a su vez han heredado el reino de la poesía de sus maestros: Octavio Paz, Rosario Castellanos, José Emilio Pacheco, José Carlos Becerra, Eduardo Lizalde, Gerardo Deniz, Francisco Hernández, Coral Bracho, Mario Santiago Papasquiaro, Ricardo Castillo.

En este número de la revista América sin Nombre, nos proponemos estudiar la poesía que se publica en los últimos dieciocho años, en el siglo XXI. En un país donde el cambio de era significa tanto, planteamos una panorámica de las líneas trazadas en lo que va de milenio. Frente a la crisis finisecular y las escenas apocalípticas que describían José Emilio Pacheco o Coral Bracho encontramos ahora textos que empiezan a ver la luz tras la dictadura perfecta de la que hablaba Vargas Llosa para referirse a las siete décadas de gobierno priísta, hasta el 30 de noviembre del año 2000. En ese año augural también surgen el Congreso de Poesía y Poética de Puebla o la Feria del Libro de la Ciudad de México, cuyo Festival Internacional de Poesía ya se encuentra afianzado. La apertura trajo consigo nuevos eventos, editoriales (Almadía, Bonobos, Era, Cal y Arena, Mantis Editores, Filodecaballos, Malpaís Ediciones, Verso destierro, Cuadrivio), talleres (similares a los del 68), poetas de México (que pueden nacer o vivir en otro país) y proyectos colectivos, como Círculo de Poesía o el Seminario de Investigación en Poesía Mexicana Contemporánea que vertebra en parte los estudios presentados en estas líneas. En 2018, fecha del cierre, se celebran los primeros cincuenta ańos del Premio Nacional de Poesía Aguascalientes, uno de los premios más prestigiosos del panorama nacional e internacional.

Cuando propusimos el título para el número 23 de la revista América sin Nombre, "Madurez de la joven poesía mexicana", nos encontramos con un problema: es que, de primeras, no se entiende. $\mathrm{Y}$ así ocurre con la lírica que se viene publicando desde Gerardo Deniz a Xitlalitl Rodríguez Mendoza.
Nuestro propósito no es explicar la poesía mexicana del tercer milenio, sino entender el conjunto de lo que se publica en la mayoría de edad del siglo XxI (2000-2018) como una diversidad encriptada por la dispersión temática y formal.

El deslumbramiento que provoca la juventud ciertamente es muy nuevo y su punto de partida puede ubicarse alrededor de los movimientos estudiantiles de 1968. En 1966, Poesía en movimiento, la selección preparada por Octavio Paz, Alí Chumacero, José Emilio Pacheco y Homero Aridjis, fue una inesperada apuesta por la poesía joven, entre el alarde propio de quien desea hacerse notar (los dos poetas más jóvenes también fungían como compiladores) y el deseo de animar a su público lector con una poesía que fuera próxima en el tiempo. La iniciativa, sin embargo, vino de más atrás; como recuerda Gabriel Zaid en su Asamblea de poetas jóvenes de México, de 1980: "A principios de 1960, la Revista Mexicana de Literatura, dirigida por Juan García Ponce y Tomás Segovia, publicó un número de "Nuevos poetas". El más joven, Homero Aridjis, no había cumplido veinte años. A mediados de 1966, Carlos Monsiváis publicó La poesía mexicana del siglo XX y el poeta más joven tenía veintiséis años: era otra vez Aridjis. A fines de 1966, Octavio Paz, Alí Chumacero, José Emilio Pacheco y Homero Aridjis publicaron Poesía en movimiento y el poeta más joven era también Aridjis» (Zaid 11). Incluso partiría de más atrás, si pensamos en el precedente de Jesús Arellano y su antología Poetas jóvenes de México (1955), también con un quinceañero Aridjis como el más joven. La idea, en todo caso, cristaliza. Siglo xxi publicaría Poesía joven de México (1967), con una selección de textos de Alejandro Aura, Leopoldo Ayala, José Carlos Becerra y Raúl Garduño que agotó su primera edición de 3000 ejemplares en 10 meses, a la que siguieron Poesía joven de Panamá (1971), una Poesía joven de Chile (1973) y una Poesía joven de Colombia (1975).

En Poesía en movimiento quedaron fuera poetas que serían fundamentales para la historia inmediata de la poesía mexicana, como Eduardo Lizalde y Gerardo Deniz. En aquel momento, tenían treinta y siete y treinta y dos años, respectivamente. Aunque por entonces Lizalde publicaba ya su cuarto poemario, Cada cosa es babel (1966), es cierto que Deniz no haría lo propio hasta Adrede (1970). Los poemas de este último todavía no aparecían más que en revistas, al igual que ocurría con Francisco Cervantes. Sin embargo, el queretano, cuatro años menor que Deniz e igualmente sin publicar, sí formó parte de Poesía en movimiento. En la actualidad, poetas 
como Karen Villeda o Christian Peña, que nacieron en 1985 , cuentan ya con numerosos libros de poesía y con sendas docenas de reconocimientos. De hecho, Peña es uno de los más jóvenes en conseguir el Premio de Poesía Aguascalientes (antes de llegar a la treintena, tras José de Jesús Sampedro, Ernesto Lumbreras, José Javier Villarreal y Alejandro Aura). Si partimos de quien lleva dieciocho años publicando poesía reconoceremos la formación, el canon y los condicionantes que se han dado para escribir de diversas maneras. La retrospectiva nos permite leer y explicar la madurez, el crecimiento, el rito de paso, de una generación de poetas jóvenes que, por varios motivos, ya no son ni generación ni jóvenes.

La teoría de las generaciones de Ortega y Gasset fue sin duda una herramienta esencial para explicar las primeras décadas del siglo xx: ahí quedan etiquetas útiles como la generación de Contemporáneos, la de Taller, la de Vuelta; pero lo cierto es que las generaciones que compartían grupo, ubicación, credo y manifiestos comunes se convirtieron paulatinamente en promociones hasta llegar a disociarse definitivamente en prácticas del siglo xxI que giran en órbitas muy distintas y distantes alrededor o en contra del Estado como apoyo público y en pos de una originalidad adánica idealizada para la que no existen fronteras expresivas infranqueables.

La creación del FONCA en 1989 prolonga la juventud que terminaba a los veintinueve (Padrón 26) y abre el camino a la profesionalización. Este fenómeno, también muy reciente, complica el entorno de la creación literaria, antes reservada para quienes podían vivir de otra cosa y se dedicaban a la poesía por vocación, al sumar muchas variantes a la ecuación del prestigio literario. Se invierten las fuerzas en un proyecto que, en el mejor de los casos, termina como libro que nadie lee; y si falta quien lea, ¿cómo valorar esa obra? La crítica debe de orientar en los demasiados libros que se publican hoy y reconducir un fenómeno complejo en la dirección no de la especulación canónica, sino del influjo que obras poderosas tienen en otros poetas. En estas páginas, sus múltiples aristas serán tratadas desde acercamientos generales que expliquen eso que «de primeras no se entiende».

¿Qué significa ser poeta joven en México? Para el Premio Nacional de Poesía Joven Elías Nandino o para el Premio Nacional de Poesía Joven Francisco Cervantes Vidal consideran «jóvenes» a poetas que tienen hasta treinta años de edad; en cambio, para el certamen de Poesía Joven Alejandro Aura no deben pasar de los veintiséis.
Gabriel Zaid planteaba una extensa y variada selva en su Asamblea de poetas jóvenes de México. Además, mostraba un problema que, difícil de resolver, buscamos replantear a partir de la misma máxima: «No hay razón alguna para suponer que esta tradición vaya a interrumpirse. La abundancia, la juventud, cierta falta de oficio, algunos cambios de gusto, la dispersión de grupos y publicaciones, hacen confuso el panorama» (23). Más recientemente, continuando con la muestra que edita Carmina Estrada, Un orbe más ancho. 40 poetas jóvenes (1971-1983) (2005), Gerardo Grande y Manuel de J. Jiménez, en Astronave. Panorámica de poesía mexicana (19851993) (2013), siguen los puntos cardinales que planteó Jorge Fernández Granados y buscan «combatir el prejuicio del "poeta joven" como un adolescente incapaz que necesita el aliento de los mayores. Pensemos más en una nueva poesía y menos en inmadurez poética» (8). Sin embargo, la tradición continúa presente en poetas que nacen al final del milenio y tratan de inaugurar un nuevo movimiento. No necesitan el aliento de los mayores, es cierto, pero sí el de la crítica.

Si para José Emilio Pacheco en su poema «Crítica de la poesía»: "nuestra época / nos dejó hablando solos» (No me preguntes cómo pasa el tiempo 36), Atardecer en los suburbios (2011), de Minerva Reynosa, incide en los cambios que acarrea el paso del tiempo; mientras que Horacio Warpola reivindica la juventud en Gestas (2015): «Estoy joven y a veces me siento como un viejo y a veces me siento / como un niño / Son los treinta -dicen- / No quiero quedarme a medias / A mi gobierno le conviene / que sea una trucha en un estanque de crianza / fácil de pescar fácil de devorar» (11). El tiempo de la poesía joven pasa muy rápido

En lo que va de milenio se han publicado algunos libros fundamentales para entender la lírica reciente. Sin canon que valga, destacan: la memoria de Los hábitos de la ceniza (2000), de Jorge Fernández Granados (Ciudad de México, 1965); la épica de Hay batallas (2005), de María Rivera (Ciudad de México, 1971); el gorjeo de Todas estas puertas (2008), de Amaranta Caballero (Guanajuato, 1973); la traducción silente de Cuaderno de los sueños (2009), de Manuel Iris (Campeche, 1983); las acumulaciones de Se llaman nebulosas (2010), de Maricela Guerrero (Ciudad de México, 1977); el filo de Sobrenaturaleza (2010), de Hernán Bravo Varela (Ciudad de México, 1979); el desplazamiento de Estilo (2011), de Dolores Dorantes (Córdoba, Veracruz, 1973); el testimonio colectivo de Antígona 
González (2012), de Sara Uribe (Querétaro, 1978); el discurso obliterado de Ruido (2012), de Alejandro Albarrán (Ciudad de México, 1985); los niveles de lectura de Álbum Iscariote (2013), de Julián Herbert (Acapulco, 1971); la cerrazón de A tiro de piedra (2014), de Daniel Téllez (Ciudad de México, 1972); la crudeza de Bitácora de mujeres extrañas (2014), de Esther M. García (Ciudad Juárez, Chihuahua, 1987); las conexiones de Nudo Vortex (2015), de Rocío Cerón (Ciudad de México, 1972); el compromiso de De la materia en forma de sonido (2015), de Óscar de Pablo (Cuernavaca, Morelos, 1979); o el diálogo ausente de Caída del búfalo sin nombre. Ensayo sobre el suicidio (2017), de Alejandro Tarrab (Ciudad de México, 1972).

En los ensayos críticos advertimos la evolución categorial de El ocaso de los poetas intelectuales y la "generación del desencanto" (2010), de Malva Flores; el tránsito social de Canibal. Apuntes sobre poesía mexicana reciente (2010), de Julián Herbert; la amplitud de Historia critica de la poesía mexicana (2015), de Rogelio Guedea; la metaliteratura de Artes poéticas mexicanas (de los Contemporáneos a la actualidad) (2015), de Carmen Alemany; la subjetividad de De la intimidad. Emociones privadas y experiencias públicas en la poesía mexicana (2016), de Luis Vicente de Aguinaga; o la metodología de Mexican Literature in Theory (2018), de Ignacio M. Sánchez Prado. Así pues, buena parte de la poesía conforma la crítica que presentamos.

Los numerosos poemarios contrastan con los escasos estudios al respecto; de manera que las antologías, por sus prólogos y por la frecuencia con que se publican y se enfrentan, ofrecen un panorama sesgado que atiende a intereses y disputas, tal como veremos en los siguientes artículos a raíz de El manantial latente. Muestra de poesía mexicana desde el ahora: 1986-2002 (2002), con la alternativa de Ernesto Lumbreras y Hernán Bravo Varela; la rigurosidad de El decir y el vértigo. Panorama de la poesía hispanoamericana reciente (1965-1979) (2005), de Rocío Cerón, Julián Herbert y León Plascencia Nol; las contradicciones de Tigre la sed. Antología de poesía mexicana contemporánea 19502005 (2006), de Víctor Manuel Mendiola, Miguel Ángel Zapata y Miguel Gomes; el personal foco de La luz que va dando nombre. Veinte años de la poesía última en México (2007), de Alí Calderón; el reclamo de Divino tesoro: muestra de nueva poesía mexicana (autores nacidos entre 1876 y 1990) (2008), de Luis Felipe Fabre; la actualización de El oro ensortijado, poesía viva de México (2009), de Mario Bojórquez,
Alí Calderón, Jorge Mendoza y Álvaro Solís; la patria de País de sombra y fuego (2010), de Jorge Esquinca; la visión restrospectiva de un proyecto público como el de 20 años de poesía, jóvenes creadores del FONCA (2010), de Jorge Fernández Granados; el Sudd de 359 Delicados (con filtro). Antología de la poesía actual en México (2012), de Pedro Serrano y Carlos López Beltrán; la exhaustividad de Antología general de la poesía mexicana. Poesía del México actual. De la segunda mitad del siglo XX a nuestros días (2014), de Juan Domingo Argüelles; o la polémica de México 20. La nouvelle poésie mexicaine (2016), de Jorge Esquinca, Tedi López Mills y Myriam Moscona. Sobre las numerosas y polémicas antologías de poesía mexicana Eva Castañeda y Alejandro Higashi dedicarán los dos números de la revista Signos Literarios en 2019.

Si los temas varían desde el intimismo a la sátira digital, con cierta vinculación al dificultismo y la poesía conversacional desde Deniz y Pacheco, respectivamente, descuellan algunas técnicas que explicitan el proceso de escritura, las numerosas referencias intertextuales y, a la vez, la inseguridad ante el texto fijo o definitivo, lo cual se opone a la activa experimentalidad y aprovecha las alternativas que ofrece el procesador de textos (Ballester \& Higashi).

Quienes nacen entre los setenta y los ochenta publican en este siglo y desde distintas regiones del país. El proceso de descentralización es lento, pues la ciudad de México acapara buena parte de actividades, becas y premios sobre poesía. Además, la madurez conlleva una emancipación y recentralización. Estos poetas que paulatinamente dejan de ser jóvenes abandonan la casa familiar para independizarse en el núcleo. Pese a la complicación de estos márgenes, iniciativas como la de Almadía y su reciente residencia en Oaxaca ofrecen alternativas.

Dónde y cuándo se advierten las consecuencias de la recién creada Academia Mexicana de Poesía; cómo influye la llegada de Visor a México, en 2017, a través de Círculo de Poesía; cuál será el nuevo rumbo del cincuentenario Premio Nacional de Poesía Aguascalientes; qué hacer cuando ya no se es joven creador; cómo afecta al poema el tránsito de la juventud a la madurez: tales serán interrogantes que nos pueden despertar las sucesivas miradas críticas.

A continuación, veremos lo que supone la reciente lírica en México desde la madurez necesaria para estudiar los últimos casos de artes poéticas, experimentación, feminismo y temas que conectan, ahora, la tradición con la herencia renovada. A sus dieciocho años, la poesía mexicana del tercer 
milenio retoma dos tradiciones: la coloquialidad de José Emilio Pacheco y el dificultismo de Gerardo Deniz. Dichas vertientes son analizadas y comparadas a voces más recientes por Ignacio Ruiz-Pérez y Alejandro Higashi, respectivamente. Por su parte, Ignacio M. Sánchez Prado estudia la crisis del lenguaje en la última poesía mexicana, a la vez que Jorge Fernández Granados va de los pioneros a los epígonos como sucesión de la tradición renovada en su continuidad antológica; mientras que Malva Flores relaciona las políticas públicas con la edición y sus numerosos condicionantes. En este contexto, Jorge Aguilera y Eva Castañeda leen desde la política de los tres últimos gobiernos, mientras que Sara Uribe observa el cuerpo, la violencia y la reescritura desde casos particulares que explican lo colectivo, al tiempo que Diana del Ángel y Mariana Ortiz registran la presencia que tienen las lenguas originarias en el género literario que nos ocupa. Si Israel Ramírez se centra en las revistas que enmarcan este complejo y variado catálogo, Alejandro Palma y Jocelyn Martínez explican la evolución de la escritura conceptual en las últimas décadas. Por último, Ignacio Ballester atiende a la poesía mexicana desde España, fijando los puntos de partida y encuentro de tal paralelismo. Los once artículos buscan ofrecer un panorama amplio del fondo y la forma que tiene la joven, mas madura, poesía mexicana.

La revista América sin Nombre vio la luz en 1999; por lo que, de alguna manera, también goza ya de mayoría de edad. Agradecemos a Carmen Alemany la confianza que nos dio para coordinar este número a través del Seminario de Investigación en Poesía Mexicana Contemporánea, a quienes debemos el incansable apoyo desde el primer momento. Igualmente, este trabajo no sería posible sin el generoso equipo de evaluación anónimo que ha evaluado cada artículo.

Resulta un riesgo estudiar lo contemporáneo, pues la falta de tiempo impide ver los cambios y las constantes que finalmente no son modas sino tradiciones que se renuevan y se asientan. Sin embargo, tal ausencia de perspectiva queda compensada por la posibilidad de entender un fenómeno complejo en el marco de un mismo contexto y de la mano de poetas, críticas y críticos coetáneos. Es esta, pues, una oportunidad para celebrar la madurez de la joven poesía mexicana a través de la autoreflexión.

\section{Bibliografía}

De Aguinaga, Luis Vicente. De la intimidad. Emociones privadas y experiencias públicas en la poesía mexicana. México: Fondo de Cultura Económica, 2016.

Albarrán, Alejandro. Ruido. México: Bonobos / Consejo Nacional para la Cultura y las Artes, 2012. Poesía Mexa. Medio de publicación (web). <https://poesiamexa.wordpress.com/2016/03/12/alejandro-albarranpolanco/>. Consultado 26 febr. 2017.

Alemany Bay, Carmen. «Novedades y regresos en la poesía hispanoamericana de las últimas décadas». Carmen Alemany Bay y Pilar Blanco (eds.). Háblame de poesía. Reflexiones sobre la poesía española e hispanoamericana y conversaciones poéticas. Alicante: Instituto Alicantino de Cultura Juan Gil-Albert, 2009: 13-28. Círculo de Poesía. Medio de publicación (web). <http:// circulodepoesia.com/2015/08/carmen-alemanybay-novedades-y-regresos-en-la-poesia-hispanoamericana-de-las-decadas-recientes/>. Consultado 26 ag. 2015.

Alemany Bay, Carmen (coord.). Artes poéticas mexicanas (de los Contemporáneos a la actualidad). Guadalajara: Centro Universitario de Ciencias Sociales y Humanidades, Universidad de Guadalajara, 2015.

Arellano, Jesús (comp.). Poetas jóvenes de México. México: Libro-Mex, 1955.

ArgǘlLes, Juan Domingo (comp.). Antología general de la poesía mexicana. Poesía del México actual. De la segunda mitad del siglo xx a nuestros días. México: Océano, 2014.

Ballester, Ignacio y Alejandro Higashi. «Tachaduras y borraduras en la poesía mexicana contemporánea (Albarrán, Herbert, Alcantar)». Signos Literarios, 25 (2017): 8-43. Signos Literarios. Medio de publicación (web). <https://signosliterarios.izt.uam.mx/index. php/SLIT/article/view/1255>. Consultado 21 febr. 2018.

Bojórquez, Mario, Alí Calderón, Jorge Mendoza y Álvaro Solís (comps.). El oro ensortijado, poesía viva de México. Puebla: Eón, 2009.

Bravo Varela, Hernán. Sobrenaturaleza. Valencia: PreTextos, 2010.

Caballero Prado, Amaranta. Todas estas puertas. México: Consejo Nacional para la Cultura y las Artes, 2008. Poesía Mexa. Medio de publicación (web). <https:// poesiamexa.wordpress.com/2016/03/19/amarantacaballero-prado/>. Consultado 8 febr. 2017.

Calderón, Alí (dir.). La luz que va dando nombre, Veinte años de la poesía última en México 1965-1985. Selección de Alí Calderón, José Antonio Escobar, Jorge Mendoza y Álvaro Solís. Puebla: Gobierno del Estado de Puebla, 2007. 
Cerón, Rocío, Julián Herbert y León Plascencia (selección y prólogo). El decir y el vértigo. Panorama de la poesía hispanoamericana reciente (1965-1979). Guadalajara: Filodecaballos / Consejo Nacional para la Cultura y las Artes, 2005.

Cerón, Rocío. Nudo Vortex. México: Edición Proyecto Literal, 2015.

Clark de Lara, Belem. "¿Generaciones o constelaciones?». Belem Clark de Lara y Elisa Guerra (coords.). La república de las letras: asomos a la cultura escrita del México decimonónico. México: Universidad Nacional Autónoma de México. 1 (2005): 11-46.

Dorantes, Dolores. Estilo. Guadalajara: Mano Santa, 2011. Issuu. Medio de publicación (web). <https:// issuu.com/luisfernandoortega/docs/dolores_dorantes>. Consultado 10 febr. 2017.

EsQuinca, Jorge (comp.). País de sombra y fuego. Prólogo de José Emilio Pacheco. Guadalajara: Maná / Universidad de Guadalajara / Selva Negra, 2010.

Esquinca, Jorge, Tedi López Mills y Myriam Moscona. México 20. La nouvelle poésie mexicaine. Prólogo de Philippe Ollé-Laprune, traducción de Jean-Luc Lacarriere y Joani Hocquenghem. Paris: Le Castor Astral / Secretaría de Cultura de México, 2016.

Estrada, Carmina (ed.). Un orbe más ancho. 40 poetas jóvenes (1971-1983). México: Universidad Nacional Autónoma de México, 2005.

Fabre, Luis Felipe (comp.). Divino tesoro: muestra de nueva poesía mexicana (autores nacidos entre 1876 y 1990). México: Fundación del Centro Histórico de la Ciudad de México, 2008.

Fernández Granados, Jorge. Los hábitos de la ceniza. México: Consejo Nacional para la Cultura y las Artes / Joaquín Mortiz, 2000.

Fernández Granados, Jorge. «Poesía Mexicana de Fin de Siglo. Para una calibración de puntos cardinales». Víctor Toledo (coord.). Poética mexicana contemporánea. Puebla: Benemérita Universidad Autónoma de Puebla, 2000: 221-239.

Fernández Granados, Jorge. 20 años de poesía. Jóvenes creadores del FONCA. México: Consejo Nacional para la Cultura y las Artes, 2010.

Flores, Malva. El ocaso de los poetas intelectuales y la "generación del desencanto". Xalapa: Universidad Veracruzana, 2010.

García, Esther M. Bitácora de mujeres extrañas. México: Consejo Nacional para la Cultura y las Artes, 2014.

Grande, Gerardo y Manuel de J. Jiménez (comps.). Astronave. Panorámica de poesía mexicana (19851993). México: Universidad Nacional Autónoma de México, 2013.
Guedea, Rogelio (coord.). Historia crítica de la poesía mexicana. 2 ts. México: Fondo de Cultura Económica / Consejo Nacional para la Cultura y las Artes, 2015.

Guerrero, Maricela. Se llaman nebulosas. México: Consejo Nacional para la Cultura y las Artes, 2010. Poesía Mexa. Medio de publicación (web). <https:// poesiamexa.wordpress.com/2016/03/19/maricelaguerrero/>. Consultado 14 febr. 2017.

Herbert, Julián. El nombre de esta casa. México: Consejo Nacional para la Cultura y las Artes, 2013 [ed. or. 1999].

Herbert, Julián. Caníbal. Apuntes sobre poesía mexicana reciente. Toluca: Bonobos, 2010. Transtierros. Medio de publicación (web). <http://transtierros.blogspot. com/2015/05/apun-tes-sobre-poesia-mexicanareciente.html>. Consultado 24 en. 2017.

Herbert, Julián. Álbum Iscariote. México: Era / Consejo Nacional para la Cultura y las Artes, 2013.

Higashi, Alejandro. «Hitos provisionales en el perfil de una generación: poetas mexicanos nacidos entre 1975 y 1985». Literatura Mexicana, 25 (2014): 49-74. Disponible en <http://www.sciencedirect.com/ science/article/pii/S0188254614726121> [última consulta: 19 de enero de 2016].

Higashi, Alejandro. PM / XXI / 360 . Crematística y estética de la poesía mexicana contemporánea en la era de la tradición de la ruptura. México: Tirant Humanidades / Universidad Autónoma Metropolitana, 2015.

Iris, Manuel. Cuaderno de los sueños. México: Consejo Nacional para la Cultura y las Artes, 2009. Disponible en <http://cl.ly/0X380Z1h3706> [última consulta: 7 de marzo de 2017].

Lumbreras, Ernesto y Hernán Bravo Varela (comps.). El manantial latente. Muestra de poesía mexicana desde el ahora: 1986-2002. México: Consejo Nacional para la Cultura y las Artes, 2002.

Mendiola, Víctor Manuel, Miguel Ángel Zapata y Miguel Gomes (comps.). Tigre la sed. Antología de poesía mexicana contemporánea 1950-2005. Madrid: Hiperión, 2006.

De Pablo, Óscar. De la materia en forma de sonido. México: Instituto Literario de Veracruz / Consejo Nacional para la Cultura y las Artes, 2015.

Pacheco, José Emilio. No me preguntes cómo pasa el tiempo. México: Joaquín Mortiz, 1969.

Pacheco, José Emilio. Desde entonces. México: Era, 1980.

Padrón Innamorato, Mauricio. «Entre la niñez y el ser adulto ¿quiénes son los jóvenes?». Encuentros 2050, 5 (mayo 2017): 26-27. Encuentros 2050. Medio de publicación (web). <https://en.wordpress.com/ typo/?subdomain $=$ encuen-tros $2050>$. Consultado 14 febr. 2018. 
Paz, Octavio, Alí Chumacero, José Emilio Pacheco y Homero Aridjis (comps.). Poesía en movimiento. México: Siglo xxi, 2008 [ed. or. 1966].

Reynosa, Minerva. Atardecer en los suburbios. México: Consejo Nacional para la Cultura y las Artes, 2011. Poesía Mexa. Medio de publicación (web). <https:// poesiamexa.files.wordpress.com/2016/03/atardeceren-los-suburbios.pdf>. Consultado 6 en. 2018.

Rivera, María. Hay batallas. México, Consejo Nacional para la Cultura y las Artes / Instituto Nacional de Bellas Artes / Joaquín Mortiz, 2005.

Rueda Olvera, Sonia. «Las demasiadas becas». Tierra Adentro, 194 (agosto 2014): 19-21.

Sánchez Prado, Ignacio M. (ed.). Mexican Literature in Theory. New York: Bloomsbury, 2018.
Serrano, Pedro y Carlos López Beltrán (comps.). 359 Delicados (con filtro). Antología de la poesía actual en México. Santiago de Chile: Ediciones LOM, 2012.

Tarrab, Alejandro. Caída del búfalo sin nombre. Ensayo sobre el suicidio. México: Malpaís Ediciones, 2017.

TÉllez, Daniel. A tiro de piedra. Toluca: Bonobos, 2014.

Uribe, Sara. Antígona González. México: Sur+, 2012. Poesía Mexa. Medio de publicación (web). <https:// poesiamexa.wordpress.com/tag/sara-uribe/ $>$. Consultado 26 febr. 2017.

Warpola, Horacio. Gestas. Querétaro: Consejo Nacional para la Cultura y las Artes / Ediciones El Humo, 2015. Poesía Mexa. Medio de publicación (web). <https:// poesiamexa.wordpress.com/2016/03/19/horaciowarpola/>. Consultado 4 mzo. 2018.

Zaid, Gabriel (comp.). Asamblea de poetas jóvenes de México. México: Siglo xxi, 1982 [ed. or. 1980]. 